\title{
Adult Retroperitoneal Mature Cystic Teratoma Masquerading as Non-Endocrine Primary Adrenal Tumor
}

\author{
Fatima Tuz Zahra Shakir, Rizwan Sultan, Riaz Siddiqui, M. Zubair Shah and Ayesha Javed \\ Department of Surgery, Islamabad Medical Complex (IMC), Nescom, Islamabad, Pakistan
}

\begin{abstract}
Teratomas belong to non-seminomatous germ cell tumors and originate from totipotent germ cells. They are mostly made up of tissues derived from all three layers, i.e., endoderm, mesoderm and ectoderm. They can be mature teratomas, which are benign or immature teratomas, having malignant potential. Extra-gonadal mature cystic teratomas are uncommon, particularly in retroperitoneum. Other sites are sacrococcygeal, mediastinal, and pineal regions. They are mostly detected in females in reproductive age group. In this case report, we discuss a female patient being worked up for persistent vomiting and food indigestion, initially managed on the line of acid peptic disease. She was found to have a large retroperitoneal mass in adrenal region producing mass effect on intestines. Later on, it was discovered to be mature cystic teratoma on histopathological evaluation.
\end{abstract}

Key Words: Mature teratoma, Adrenal gland, Retroperitoneum.

How to cite this article: Shakir FTZ, Sultan R, Siddiqui R, Shah MZ, Javed A. Adult Retroperitoneal Mature Cystic Teratoma Masquerading as Non-Endocrine Primary Adrenal Tumor. J Coll Physicians Surg Pak 2021; 31(11):1351-1353.

\section{INTRODUCTION}

Teratomas belong to non-seminomatous germ cell tumors and originate from totipotent germ cells. The name is derived from the Greek word meaning 'monster tumor, because mature teratoma is composed of a mixture of bone, cartilage, teeth, hair and squamous epithelium. They arise from totipotent cells with multi-directional potential of differentiation to different types of somatic cells. They most commonly occur in sacrococcygeal $(57 \%)$ region in newborns and in gonads $(29 \%)$ in adults; however, they can occur at other rare sites, especially retroperitoneal region (4\%), ${ }^{1,2}$ Primary adrenal teratomas are exceedingly rare. We herein present a case of a young lady who initially presented with dyspeptic symptoms, but was later found to be a case of mature cystic teratoma of presumably left adrenal gland.

\section{CASE REPORT}

A 31-year married female with five children and no previous comorbids, presented to general practitioner with complaints of food indigestion and multiple episodes of vomiting after food intake. Vomiting was occasionally associated with mild left-sided upper abdominal pain but never severe enough to present in emergency room.

Correspondence to: Dr. Fatima Tuz Zahra Shakir, Department of Surgery, Islamabad Medical Complex (IMC),

Nescom, Islamabad, Pakistan

E-mail: fatimatuzahra04@gmail.com

Received: July 21, 2020; Revised: September 01, 2020;

Accepted: September 29, 2020

DOI: https://doi.org/10.29271/jcpsp.2021.11.1351
She was under workup for acid peptic disease and had routine ultrasound, which revealed a cystic mass measuring $9.4 \times 4.2$ $\mathrm{cm}$ in left hypochondrium. CT scan showed a mass with fat, fluid, calcific densities in left lumbar region, anteromedial to left kidney. It measured $7.9 \times 7.9 \times 9.7 \mathrm{~cm}$. Left kidney was pushed posteriorly but fat planes between mass and kidney were intact. It was abutting left renal vein and a small segment of jejunum (Figure 1). Left adrenal gland was not seen separate from the mass.

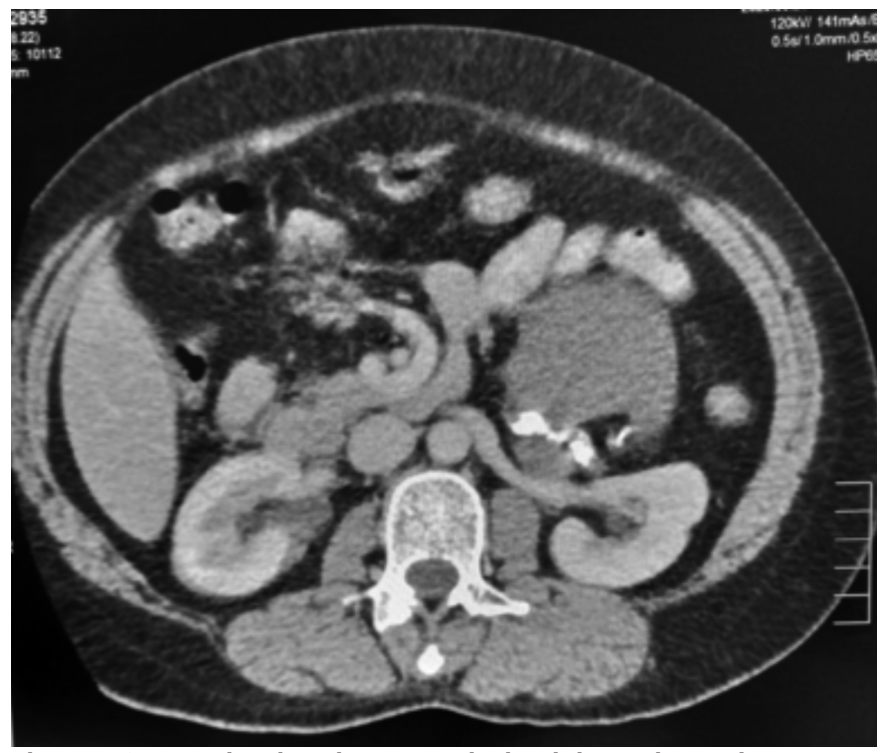

Figure 1: CT scan showing a large mass in the abdomen located anteromedial to the left kidney.

On examination, the patient was vitally stable. Abdomen was soft and non-tender and the mass was palpable on deep palpa- 
tion in the left lumber region. Bowel sounds were normal on auscultation. All laboratory workups were normal. Patient was scheduled for exploratory laparotomy for mass excision. Peroperative findings showed a large mass with both cystic and solid composition, replacing anterior aspect of Gerota's fascia and in close proximity of renal vein. Adrenal gland could not be identified on left side. Left kidney was easily separable from the lesion. The mass was excised in toto (Figure 2).

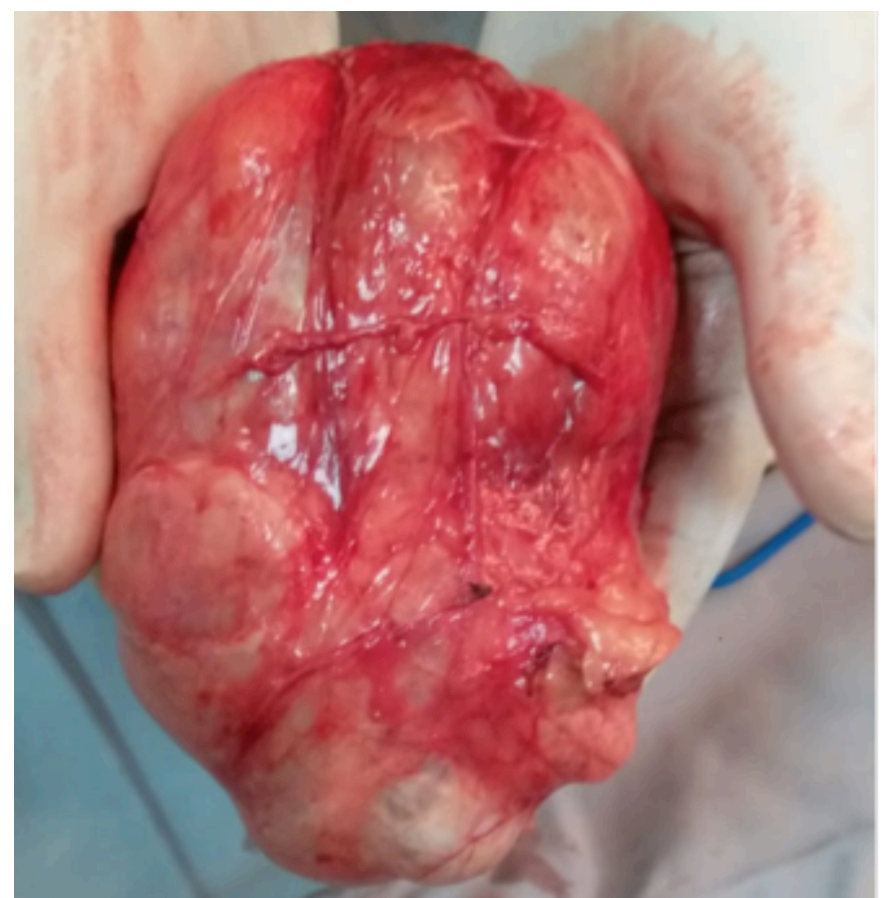

Figure 2: Gross appearance of the intact excised mass lesion.

Post operatively, the patient remained stable and was discharged home after three days.

Histopathology revealed tan white mucoid cystic material and calcified solid material on gross examination. Microscopically, it had mixture of tissues from all three germinative layers. Hair structures, mature glial tissue, and calcification with mature cartilage were identified. Respiratory epithelium and mucinous glands were also present. No immature component was found and the diagnosis of mature cystic teratoma of presumably left adrenal gland was suggested. Since, normal adrenal tissue was not identified, it was assumed that the tumor had completely replaced the native left adrenal gland.

\section{DISCUSSION}

Retroperitoneal tumors are rare having an incidence of only 0.2 to $0.8 \%$ of all tumors. Among such tumors, differential diagnosis of teratomas in retroperitoneum ranges from 6 to 18\%, and in adults, its presentation is highly unusual. Benign adrenal teratomas are mostly clinically silent throughout their development and only become apparent when they start to produce mass effect on surrounding structures. They also pose diagnostic challenge radiologically, because they mimic other adrenal tumors like angiomyolipoma, myelolipoma or liposarcoma, and rarely, even pheochromocytoma. CT scans are very helpful in guiding towards differential diagnosis, but confirmation can only be achieved by histopathology.

Li et al. in 2015 reported in their study that literature review retrieved a total of 11 cases only from PubMed in the past 10 years, with seven cases in adults, including one male and six females, aged from 21 to 64 years. ${ }^{3}$ Li et al. also studied retrospectively primary mature cystic teratoma of adrenal gland in their patients. They analysed the detailed clinical data of five patients with primary adrenal teratomas treated at Peking Union Medical College Hospital. They followed up their patients for 4-60 months and reported no recurrence of tumor.

Ramakant et al. in 2018, also reported that just eight cases of mature cystic teratoma of adrenal gland have been reported in adults in the past 10 years. ${ }^{4}$

Malignant transformation of a mature cystic teratoma is extremely uncommon. Literature has only three documented cases with an incidence of merely $0.17-2 \%$. Mandal et al. reported fourth case of malignant transformation in a mature teratoma of ovary with metastatic deposits in the omentum with very poor prognosis. ${ }^{5}$ Thus, early detection of this disease is absolutely necessary.

In conclusion, mature cystic teratoma in the adrenal gland is a lesion. Limited available data suggests that treatment comprises only compelete surgical resection of the tumor; and prognosis is good for benign mature cystic teratoma. Information regarding the progression of mature cystic teratoma is very scarce, especially lifelong outcome after its removal. Hence, further studies are required to understand the course and management of this disease.

\section{PATIENT'S CONSENT:}

Informed consent was taken from the patient for publication of this case report.

\section{CONFLICT OF INTEREST:}

The authors declared no conflict of interest.

\section{AUTHORS' CONTRIBUTION:}

FTZS, RS: Conception and design of the work; acquisition, analysis and interpretation of data for the work; drafted the work and revised it critically for important intellectual content; finally approved the version to be published.

RS, MZS, AJ: Finally approved the version to be published.

All authors agreed to be accountable for all aspects of the work in ensuring that questions related to the accuracy or integrity of any part of the work are appropriately investigated and resolved.

\section{REFERENCES}

1. Bhatti A, Al-Hindi H, Azzam A, Amin T, Abu-Zaid A. Mature (benign) cystic retroperitoneal teratoma involving the left adrenal gland in a 22-year-old male: A case report and literature review. Case Rep Oncol Med 2013; 2013:610280. doi: 10.1155/2013/610280.

2. Pandit N, Awale L, Jaiswal LS. Giant calcified retroperitoneal teratoma. Indian J Surg Oncol 2018; 9(3):436-7. doi: 
10.1007/ s13193-018-0789-8.

3. Li S, Li H, Ji Z, Yan W, Zhang Y. Primary adrenal teratoma: Clinical characteristics and retroperitoneal laparoscopic resection in five adults. Oncol Lett 2015; 10(5):2865-70. doi: 10.3892/ol.2015.3701.

4. Ramakant P, Rana C, Singh KR, Mishra A. Primary adrenal teratoma: An unusual tumor - Challenges in diagnosis and surgical management. J Postgrad Med 2018; 64(2):112. doi: 10.4103/jpgm.JPGM_588_16.

5. Mandal S, Badhe BA. Malignant transformation in a mature teratoma with metastatic deposits in the omentum: A case report. Case Rep Pathol 2012; 2012:568062. doi: 10.1155/ 2012/568062. 